\title{
Phosphorylation of different tau sites during progression of Alzheimer's disease
}

\author{
Joerg Neddens', Magdalena Temmel ${ }^{1}$, Stefanie Flunkert ${ }^{1}$, Bianca Kerschbaumer ${ }^{1,2}$, Christina Hoeller ${ }^{1,3}$, \\ Tina Loeffler ${ }^{1}$, Vera Niederkofler ${ }^{1}$, Guenther Daum², Johannes Attems ${ }^{4}$ and Birgit Hutter-Paier ${ }^{1 *}$ (D)
}

\begin{abstract}
Alzheimer's disease is characterized by accumulation of amyloid plaques and tau aggregates in several cortical brain regions. Tau phosphorylation causes formation of neurofibrillary tangles and neuropil threads. Phosphorylation at tau Ser202/Thr205 is well characterized since labeling of this site is used to assign Braak stage based on occurrence of neurofibrillary tangles. Only little is known about the spatial and temporal phosphorylation profile of other phosphorylated tau (ptau) sites. Here, we investigate total tau and ptau at residues Tyr18, Ser199, Ser202/Thr205, Thr231, Ser262, Ser396, Ser422 as well as amyloid- $\beta$ plaques in human brain tissue of AD patients and controls. Allo- and isocortical brain regions were evaluated applying rater-independent automated quantification based on digital image analysis. We found that the level of ptau at several residues, like Ser199, Ser202/Thr205, and Ser422 was similar in healthy controls and Braak stages I to IV but was increased in Braak stage VNI throughout the entire isocortex and transentorhinal cortex. Quantification of ThioS-stained plaques showed a similar pattern. Only tau phosphorylation at Tyr18 and Thr231 was already significantly increased in the transentorhinal region at Braak stage III/IV and hence showed a progressive increase with increasing Braak stages. Additionally, the increase in phosphorylation relative to controls was highest at Tyr18, Thr231 and Ser199. By contrast, Ser396 tau and Ser262 tau showed only a weak phosphorylation in all analyzed brain regions and only minor progression. Our results suggest that the ptau burden in the isocortex is comparable between all analyzed ptau sites when using a quantitative approach while levels of ptau at Tyr18 or Thr231 in the transentorhinal region are different between all Braak stages. Hence these sites could be crucial in the pathogenesis of AD already at early stages and therefore represent putative novel therapeutic targets.
\end{abstract}

Keywords: Microtubule-associated protein tau, Phosphorylation, Cingulate, Frontal, Occipital and temporal cortex, Transentorhinal region, Immunofluorescent labeling

\section{Introduction}

Alzheimer's disease (AD) is neuropathologically characterized by two hallmark lesions, which are extracellular amyloid- $\beta$ (A $\beta)$ plaques and intracellular accumulations of abnormally phosphorylated tau. $A \beta$ plaques initially develop in neocortical regions and then progress to the limbic system, subcortical nuclei and reach the cerebellum at late stages of the disease [41]. Tau pathology manifests as neurofibrillary tangles (NFTs) and neuropil threads (NTs) and primarily accumulates in the entorhinal region and subsequently progresses to the limbic system and

\footnotetext{
* Correspondence: birgit.hutter-paier@qps.com

${ }^{1}$ QPS Austria GmbH, Neuropharmacology, Parkring 12, 8074 Grambach, Austria

Full list of author information is available at the end of the article
}

neocortical regions as reflected by NFT Braak stages [8]. Tau aggregation depends on several posttranslational modifications, including but not limited to, truncation, acetylation, ubiquitination, sumoylation and phosphorylation [13, 29, 34]. The best analyzed posttranslational modification in $\mathrm{AD}$ is abnormal phosphorylation of tau which in $\mathrm{AD}$ is referred to as hyperphosphorylation and that is characterized by an at least 3-fold increase of tau phosphorylation relative to controls. Over 70 potential tau phosphorylation (ptau) sites spanning almost the entire protein structure and including some phosphorylation sites are assumed to be pathologically relevant [40]. Some of these ptau sites are known to be abnormally phosphorylated in paired helical filaments (PHFs), NFTs or NTs during progression of AD but are not

(C) The Author(s). 2018 Open Access This article is distributed under the terms of the Creative Commons Attribution 4.0 International License (http://creativecommons.org/licenses/by/4.0/), which permits unrestricted use, distribution, and 
phosphorylated in healthy brains $[10,15,22,26,28]$. Several of these ptau sites are also phosphorylated in the fetal brain and are thus associated with embryonic brain developmental processes $[10,15,22]$. In the diseased brain, ptau sites are commonly associated with tau aggregation processes such as incomplete binding and destabilization of microtubules, causing the transition from pre-tangles to NFT formation [12, 16, 21, 28, 32].

The temporal and spatial phosphorylation pattern of tau residue Ser202/Thr205 has already been well characterized since staging of $\mathrm{AD}$ cases is based on labeling of ptau Ser202/Thr205 using the specific antibody AT8 [1, $9,20,46]$. However, the phosphorylation profile of other ptau sites known to be involved in AD pathology is so far strongly neglected and analyzed only by ELISA and dot blots of AD brain tissue [20,46].

Phosphorylation at several residues during disease progression has been repeatedly investigated in tau animal models but barely in AD brain tissue [4, 7, 14, 20, 46]. Hence, the present study aims to evaluate the spatial and temporal phosphorylation profile of different tau sites by applying rater-independent automated quantification based on digital image analysis. We therefore quantified the expression of total tau, amyloid- $\beta$ plaques and the phosphorylation of seven different tau sites (Tyr18, Ser199, Ser202/Thr205, Thr231, Ser262, Ser396, Ser422) in iso- and allocortical brain regions of AD cases and controls.

\section{Materials and methods}

Human brain samples

Paraffin sections of $6 \mu \mathrm{m}$ thickness and frozen samples from AD patients of three different disease stages [Braak stages I/II, III/IV and $V / \mathrm{VI}$; [9]] and control individuals with Braak stage 0 were provided by the Newcastle Brain Tissue Resource (NBTR), Newcastle University, UK in accordance with the approval of the joint Ethics Committee of Newcastle and North Tyneside Health Authority and following NBTR brain banking procedures. The neuropathological diagnosis was performed according to

Table 1 Individual case information. Demographic patient information

\begin{tabular}{|c|c|c|c|c|c|c|c|c|}
\hline & Case & Age & Sex & Braakstage & $\begin{array}{l}\text { Post mortem delay to } \\
\text { fixation in hours }\end{array}$ & $\begin{array}{l}\text { Fixation time in } \\
\text { weeks }\end{array}$ & Clinical information & $\begin{array}{l}\text { \# on Western } \\
\text { blot }\end{array}$ \\
\hline \multirow[t]{6}{*}{ Controls } & 1 & 68 & $M$ & 0 & 54 & 7 & Cognitively normal & 1 \\
\hline & 2 & 55 & M & 0 & 41 & 11 & Cognitively normal & 2 \\
\hline & 3 & 70 & M & 0 & 72 & 6 & Cognitively normal & - \\
\hline & 4 & 78 & $\mathrm{~F}$ & 0 & 34 & 8 & Cognitively normal & 4 \\
\hline & 5 & 73 & M & 0 & 25 & 9 & Cognitively normal & - \\
\hline & mean & 68.8 & & 0 & 45.2 & 8.2 & & \\
\hline \multirow{6}{*}{$\begin{array}{l}\text { Braak I/II } \\
\text { Low }\end{array}$} & 6 & 96 & $\mathrm{~F}$ & 2 & 114 & 49 & Mild dementia & 6 \\
\hline & 7 & 77 & M & 2 & 83 & 15 & Cognitively normal & 7 \\
\hline & 8 & 94 & $\mathrm{~F}$ & 2 & 15 & 9 & Cognitively normal & - \\
\hline & 9 & 70 & M & 2 & 39 & 7 & $\begin{array}{l}\text { Multiple psychiatric and physical } \\
\text { problems }\end{array}$ & 9 \\
\hline & 10 & 74 & $\mathrm{~F}$ & 1 & 49 & 10 & Cognitively normal & 10 \\
\hline & mean & 82.2 & & 1.8 & 60 & 18 & & \\
\hline \multirow{6}{*}{$\begin{array}{l}\text { Braak III/IV } \\
\text { Mod. }\end{array}$} & 11 & 75 & M & 4 & 82 & 23 & Cognitively normal & 11 \\
\hline & 12 & 79 & M & 3 & 13 & 15 & Cognitively normal & 12 \\
\hline & 13 & 81 & M & 3 & 82 & 8 & Unspecified dementia & 13 \\
\hline & 14 & 98 & $\mathrm{~F}$ & 3 & 59 & 8 & Cognitively normal & 14 \\
\hline & 15 & 91 & M & 3 & 48 & 9 & $\begin{array}{l}\text { Moderate cognitive impairment and } \\
\text { vascular disease }\end{array}$ & 15 \\
\hline & mean & 84.8 & & 3.2 & 56.8 & 12.6 & & \\
\hline \multirow[t]{6}{*}{$\begin{array}{l}\text { Braak VNI } \\
\text { High }\end{array}$} & 16 & 84 & $\mathrm{~F}$ & 6 & 47 & 16 & $\begin{array}{l}\text { Severe dementia, anxiety and } \\
\text { depression }\end{array}$ & - \\
\hline & 17 & 77 & $\mathrm{~F}$ & 6 & 63 & 5 & Dementia & 17 \\
\hline & 18 & 80 & $\mathrm{~F}$ & 6 & 32 & 16 & Dementia & 18 \\
\hline & 19 & 86 & $\mathrm{~F}$ & 6 & 5 & 6 & Dementia & 19 \\
\hline & 20 & 89 & $\mathrm{~F}$ & 6 & 85 & 8 & Dementia & - \\
\hline & mean & 83.2 & & 6 & 46.4 & 10.2 & & \\
\hline
\end{tabular}


internationally accepted criteria [31] (Table 1). Tissue of one patient of Braak stage V/VI yielded clearly stronger pathology compared to the other cases, representing an overshoot outlier. Data of this patient were excluded for pThr231 calculations. For histological analyses, five cases per group were investigated using sections of 5 brain regions per individual: temporal cortex (TeCtx), frontal cortex (FrCtx), cingulate cortex (CiCtx), occipital cortex (OcCtx), and transentorhinal region (TEntR). All histological assessments were done blinded to clinical and neuropathological data.

\section{Immunofluorescent labeling}

Sections were deparaffinized for $10 \mathrm{~min}$ in Tissue Clear (Sakura, 1466, Netherlands) and 5 min in Tissue Clear/ $100 \%$ ethanol, washed for $5 \mathrm{~min}$ in $100 \%$ ethanol and then subsequently rehydrated with decreasing alcohol concentrations (96, 70 and 50\% ethanol for 2 min each). Thereafter, sections were washed twice for $5 \mathrm{~min}$ in PBS. Sections for labeling of human total tau, ThioflavinS (ThioS), pThr231 tau, pSer199 tau, pTyr18 tau and pSer396 tau were treated for 15 min with citrate buffer (Thermo Scientific, AP-9003) at $95{ }^{\circ} \mathrm{C}$ in a steamer and cooled down to room temperature (RT) for another $15 \mathrm{~min}$. For ThioS staining, sections were then labeled with $0.5 \%$ ThioS (Sigma-Aldrich, T1892) for $7 \mathrm{~min}$ under light protection. Afterwards, sections were pretreated with ice-cold sodium borohydride/PBS solution ( $1 \mathrm{mg} / \mathrm{ml}$; Sigma-Aldrich, 213,462) for $4 \mathrm{~min}$ and tissue was then permeabilized with $1 \%$ Triton $^{\circ} \mathrm{X}-100 / \mathrm{PBS}$ (AppliChem, A1388) for $10 \mathrm{~min}$. Non-specific labeling was blocked by incubating sections for 30-60 min either with $10 \%$ donkey serum/PBS or $10 \%$ horse serum/PBS. For antigen detection, sections were incubated with primary antibodies (Table 2) in a damp chamber. Primary antibody binding was visualized by incubating sections with secondary fluorophore conjugated antibodies (Table 3) for $60 \mathrm{~min}$. Furthermore, cell nuclei of tissue labeled for pSer199 tau, pSer396 tau and pSer422 tau were visualized by counterstaining with 4',6-Diamidin-2-phenylindol-working solution (DAPI, AppliChem, A1001) for
15 min, differentiated with $70 \%$ ethanol and washed for 5 min in PBS. Finally, sections were washed in $d_{d d} \mathrm{H}_{2} \mathrm{O}$ and covered with Moviol and coverslips.

In order to use the human sections efficiently, double labelings were performed: pSer202/Thr205 combined with pSer262 (Additional file 1: Online Source 9); ThioS combined with HT7; pSer199 combined with pSer396. Labelings against pTyr18, pThr231 and pSer422 were performed without co-labeling of another ptau residue.

\section{Imaging and image analysis}

Of each labeled brain section two mosaic images with a size of approximately $3 \mathrm{~mm}^{2}$ including white and grey matter were captured at different $\mathrm{z}$-levels and finally projected to $2 \mathrm{D}$.

Imaging of immunofluorescent labelings of pSer202/ Thr205 tau, pSer199 tau, pSer396 tau, pSer262 tau and pSer422 tau was performed using a Nikon Eclipse E400 microscope with a high aperture lens $(20 \times$ lens, numerical aperture $0.5,1 \times$ optocoupler), equipped with a Nikon DS-Qi1MC camera and controlled by the Nikon NIS-Elements AR software. For quantification of total human tau, pThr231 tau, pTyr18 tau and ThioS images were recorded using a Zeiss AxioImager.Z1 microscope with a high aperture lens and an AxioVision 4.8 software-driven AxioCam MRm digital camera (20x lens, numeric aperture $0.8,1 \times$ optocoupler).

Quantitative image analysis was performed using Image-Pro Plus (version 6.2, Media Cybernetics, Inc., Rockville, USA). Grey-scale single channel images were corrected for background intensities using lowpass filtering, and signal from autofluorescent objects (mostly lipofuscin and erythrocytes) was subtracted from the channel used for immunofluorescent labeling. Objects were identified by a combination of Edge+ filter, adequate thresholding, and size and shape restrictions (Additional file 1: Online Source 10). After defining the parameters for detecting the targeted objects, macro-driven quantitative image analysis ran automatically using the same parameters on all images. The

Table 2 List of primary antibodies for histology

\begin{tabular}{lllllll}
\hline Antigen & Clone & Species & Source & Order \# & Dilution & Incubation time \\
\hline Total human tau & HT7 & mouse & Pierce Biotechnology, USA & MN1000 & $1: 500$ & overnight at $4{ }^{\circ} \mathrm{C}$ \\
pThr231 & AT180 & mouse & Pierce Biotechnology, USA & MN1040 & $1: 200$ & overnight at $4{ }^{\circ} \mathrm{C}$ \\
pSer202+ pThr205 & AT8 & mouse & Thermo Scientific, USA & MN1020 & $1: 200$ & overnight at $4{ }^{\circ} \mathrm{C}$ \\
pSer199 & polyclonal & rabbit & Invitrogen Corporation, USA & 44-734G & $1: 500$ & $1 \mathrm{~h}$ at RT \\
pTyr18 & 9G3 & mouse & MédiMabs, Canada & MM-0194-P & $1: 1000$ & $1 \mathrm{~h}$ at RT \\
pSer396 & PHF13 & mouse & Cell Signaling Technology, USA & 9632 & $1: 500$ & $1 \mathrm{~h}$ at RT \\
pSer262 & polyclonal & rabbit & MBL International, USA & AT-5020 & $1: 250$ & overnight at 4 ${ }^{\circ} \mathrm{C}$ \\
pSer422 & 5.6 .11 & mouse & Roche Diagnostic, Switzerland & N/A & $1: 800$ & overnight at 4 ${ }^{\circ} \mathrm{C}$ \\
\hline
\end{tabular}


Table 3 List of secondary antibodies

\begin{tabular}{|c|c|c|c|c|}
\hline Antibody & Conjugation & Source & Order \# & To visualize \\
\hline \multirow[t]{3}{*}{ Donkey Anti-Mouse IgG H\&L } & AlexaFluor 555 & abcam, UK & ab150110 & pSer422 tau \\
\hline & & & & pSer396 tau \\
\hline & & & & pSer202/ pThr205 tau \\
\hline \multirow[t]{2}{*}{ Donkey Anti-Rabbit lgG H\&L } & DyLight 650 & abcam, UK & ab96922 & pSer199 tau \\
\hline & & & & pSer262 tau \\
\hline Donkey Anti-Mouse IgG H\&L & DyLight 550 & abcam, UK & ab98795 & pThr231 tau \\
\hline Donkey Anti-Mouse IgG $(H+L)$ & Cyanine Cy3 & Jackson ImmunoResearch, USA & 715-165-151 & Total human tau \\
\hline Donkey Anti-Mouse IgG H\&L & DyLight 650 & abcam, UK & ab98797 & pTyr18 tau \\
\hline Dilution of all secondary antibodi & 1:500 & & & \\
\hline
\end{tabular}

quantitative results are therefore unbiased, rater-independent and fully reproducible.

\section{Preparation of sarcosyl insoluble fraction from frozen samples}

Frozen transentorhinal cortex tissue was homogenized in $9 \mu \mathrm{l}$ per mg of cold extraction buffer 1 (25 mM Tris $\mathrm{HCl} \mathrm{pH}=7.4,150 \mathrm{mM} \mathrm{NaCl}, 1 \mathrm{mM}$ EDTA, $1 \mathrm{mM}$ EGTA, $10 \mathrm{mM}$ ß-glycerophosphate, $30 \mathrm{mM} \mathrm{NaF}, 2 \mathrm{mM}$ Na3VO4, protease and phosphatase inhibitor cocktails) and centrifuged at $80,000 \mathrm{~g}$ for $15 \mathrm{~min}$ at $4{ }^{\circ} \mathrm{C}$. The pellet was resuspended in extraction buffer $2(10 \mathrm{mM}$ Tris $\mathrm{HCl} \mathrm{pH}=7.4,800 \mathrm{mM} \mathrm{NaCl}, 300 \mathrm{mM}$ sucrose, $1 \mathrm{mM}$ EGTA, protease and phosphatase inhibitor cocktails) and centrifuged at $4000 \mathrm{~g}$ for $10 \mathrm{~min}$ at $4{ }^{\circ} \mathrm{C}$. The Supernatant was transferred to a fresh tube and sarcosyl (30\% aqueous solution) was added to a final concentration of $1 \%$ and incubated for $1.5 \mathrm{~h}$ at room temperature. After centrifugation at $80,000 \mathrm{~g}$ for $30 \mathrm{~min}$ at $4{ }^{\circ} \mathrm{C}$, the supernatant was discarded and the pellet resuspended in buffer $3(50 \mathrm{mM}$ Tris- $\mathrm{HCl}, \mathrm{pH}=7.4)$. The suspension was aliquoted and frozen until used for Western blotting.

\section{Western blot analyses}

Equal amounts of proteins of each brain extract were separated by molecular weight on a SDS-PAGE polyacrylamide gel. A protein marker visualized correct separation of the proteins and confirmed the correct protein band size. Subsequently, proteins were transferred onto a $0.45 \mu \mathrm{m}$ nitrocellulose membrane using a wet blot chamber (Bio-Rad, Hercules, USA), blocked with 5\% non-fat dry milk in $1 \times$ TBS and incubated with primary antibodies overnight. To blot for total tau, pTyr18, and pSer199 the same primary antibodies as used for histological labeling were used in a concentration of 1:1000 (Table 2). Antibodies AT180 and AT8 to label pThr231 and pSer202/Thr205, respectively, did not give any signal in the Western blot and were thus substituted by a rabbit polyclonal antibody against pThr231 (1:1000; Signalway, SAB1110-2, College Park, USA) and a rabbit monoclonal antibody, clone EPR2402, against pSer202/Thr205 (1:3000; abcam, ab108387, Cambridge, UK). All primary antibodies were incubated for $2 \mathrm{~h}$ at room temperature. Afterwards, membranes were washed in TBS and incubated in horseradish peroxidase-coupled secondary antibodies for $1 \mathrm{~h}$ at room temperature (1:5000; donkey-anti-rabbit IgG: NA934/ GENA934; sheep-anti-mouse IgG: NXA931; GE-Healthcare, Little Chalfont, UK). Proteins were detected using Wester-Bright ECL spray (Advansta, Menlo Park, USA) and placed on a X-ray film. As loading control a rabbit polyclonal GAPDH antibody (Sigma-Aldrich, G9545, St. Louis, USA) was co-labeled on each blot. To blot with 6 different antibodies, three blots were used to prevent false signals due to stripping, reblotting or blocking of antibody binding by previously used antibody. For each of the three blots, GAPDH as loading control is shown. Densitometry of all bands was performed using Image J software. Cases $3,5,8,16$ and 20 according to Table 1 are missing in this analysis since no frozen tissue was available from the corresponding brain region.

\section{Statistical analyses}

All statistical analyses and preparation of graphs were conducted using Graph-Pad Prism (version 4.03, San Diego, CA, USA). Descriptive statistical analyses were performed on all evaluated parameters including the evaluation of normal distribution using the Kolmogorov-Smirnov test.

Group variances were calculated either by one-way or two-way ANOVA. If a significant interaction among groups was detected, Newman-Keuls or Bonferroni's post-hoc analysis was followed. A detailed description of performed statistical analyses is given in the appropriate figure legend. Data were averaged and represented as mean + standard error of mean (SEM). An $\alpha$-error level of $p<0.05$ was considered significant.

\section{Results}

In order to evaluate the overall human tau expression levels, brain tissues were labeled with the anti-human 

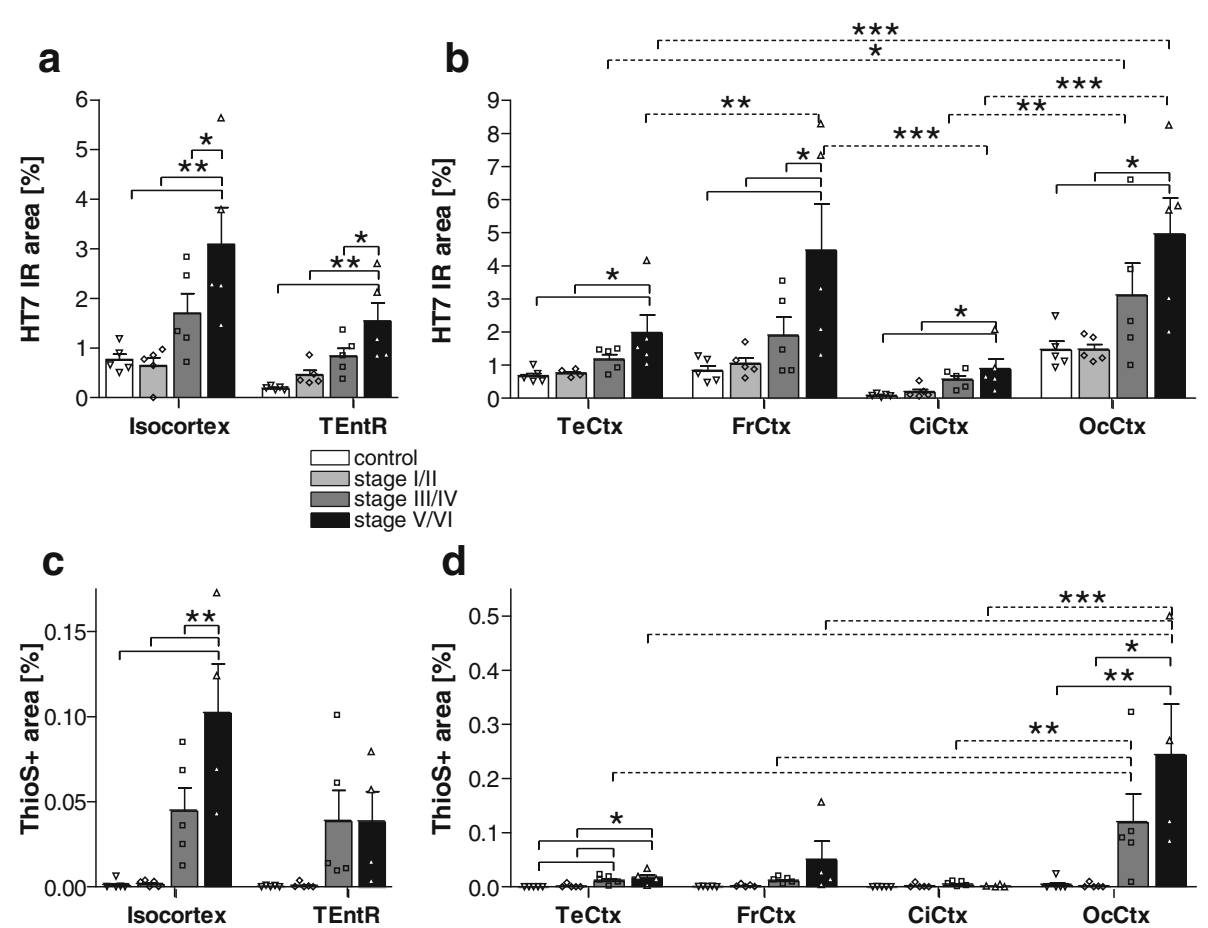

Fig. 1 Quantification of total human tau and plaque load in the cortex of AD cases. a Total human tau (HT7 antibody) immunoreactive area in percent in the isocortex and TEntR (allocortex) of different AD stages. $\mathbf{c} \beta$ sheet (ThioflavinS staining) positive area in percent in the isocortex and TEntR of different AD stages. Isocortical data in $\mathbf{a}$ and $\mathbf{c}$ present mean of different cortical regions that are shown separately in $\mathbf{b}$ and $\mathbf{d}$, respectively. Two way ANOVA followed by Bonferroni's posthoc test. Mean $+\mathrm{SEM} ; N=5 .{ }^{*} p<0.05 ;{ }^{* *} p<0.01 ;{ }^{* * *} p<0.001$. c, d One outlier of Braak stage VNI excluded for all ThioS labelings. Solid lines: Comparison of AD stages within a brain region; dotted lines: comparison of brain regions of the same AD Braak stage. CiCtx: cingulate cortex; FrCtx: frontal cortex; OcCtx: occipital cortex; TeCtx: temporal cortex; TEntR: transentorhinal region

total tau HT7 antibody. Quantification of the HT7 immunoreactive area in the isocortex and transentorhinal region (TEntR) revealed a progressive increase of total tau with advancing Braak stages. This increase in total tau signal was significant at Braak stage V/VI compared to healthy controls but also compared to both lower Braak stages I/II and III/IV (Fig. 1a). Data of the isocortex in Fig. 1a are the mean of four distinct isocortical regions, namely temporal, frontal, cingulate and occipital cortex. Separate presentation of these brain regions shows that total tau expression levels were very low in the cingulate cortex but relatively high in the occipital cortex of healthy controls (Fig. 1b). In all isocortical regions total tau levels started to increase at Braak stage III/IV and were significantly higher at Braak stage V/VI compared to controls or Braak stage I/II. At Braak stage V/VI total tau levels were highest in the frontal and occipital cortex (Fig. 1b). Since amyloid- $\beta$ plaques are, next to tau, a major pathological hallmark of AD, the ThioflavinS (ThioS) stained area was quantified in the isocortex and TEntR of AD patients and healthy controls (Fig. 1c). Threshold-based identification of ThioS staining combined with a size restriction filter enabled automatic quantification of plaque cores while excluding NFTs (Additional file 1: Online Source 11). We found that ThioS signal was significantly increased in the isocortex of Braak stage V/VI patients compared to controls and all other Braak stages; a first non-significant increase in the isocortical ThioS signal could be observed at Braak stage III/IV (Fig. 1c). In the TEntR, ThioS signal slightly increased at Braak stage III/IV but did not further increase at Braak V/VI. Almost no ThioS signal could be observed in the cingulate cortex. In the temporal and frontal cortex the ThioS signal was very low while only in the occipital cortex the ThioS signal was well measurable and progressively increasing starting at Braak stage III/IV (Fig. 1d).

After evaluating the overall total tau and plaque load, we measured the phosphorylation levels of ptau at seven different residues. First, we show phosphorylation of pSer202/Thr205 tau since this phosphorylation site is already well characterized and thus can be utilized to validate the results.

\section{pSer202/Thr205 tau}

Phosphorylation of tau at Ser202/Thr205 was low in all isocortical brain regions up to Braak stage III/IV. Only at 

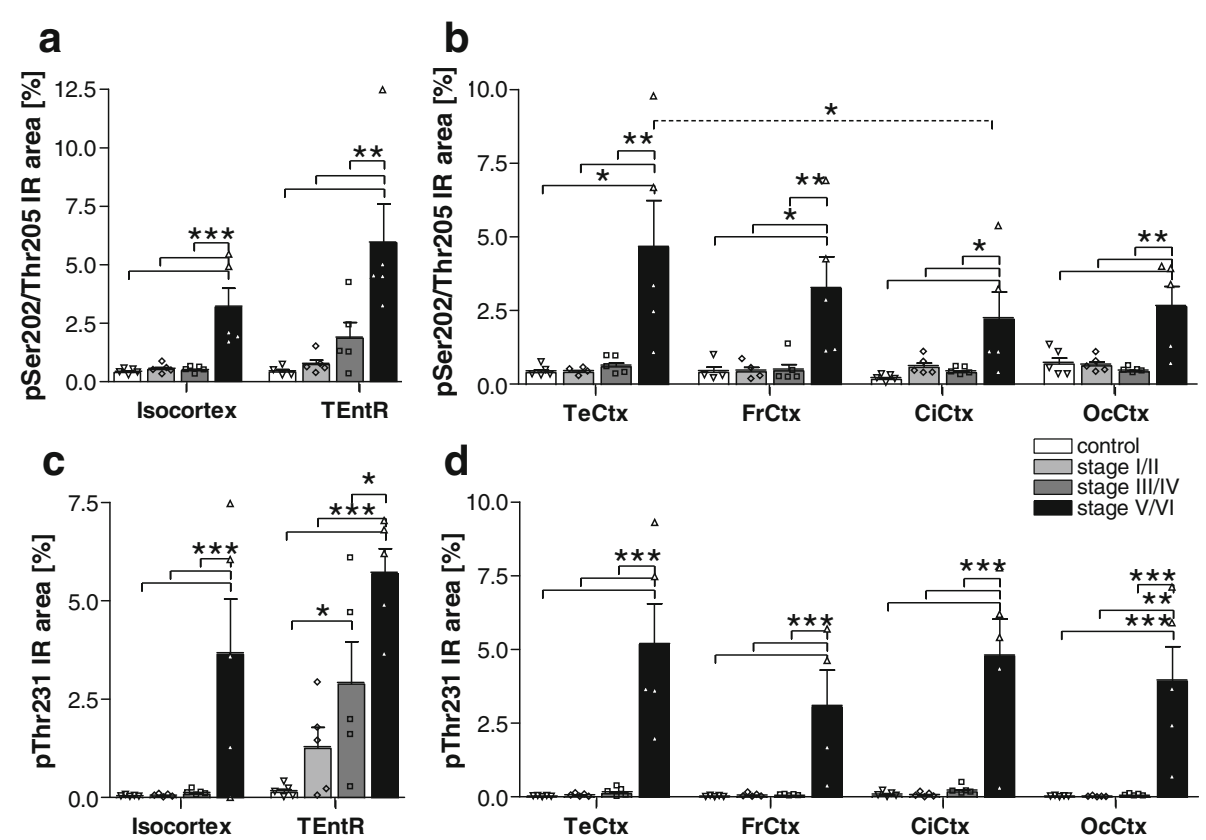

Fig. 2 Quantification of tau phosphorylation at pSer202/Thr205 and pThr231 in the cortex of AD cases. a Human pSer202/Thr205 tau immunoreactive area in percent in the isocortex and TEntR (allocortex) of different AD stages. c Human pThr231 tau immunoreactive area in percent in the isocortex and TEntR of different AD stages. Isocortical data in $\mathbf{a}$ and $\mathbf{c}$ present mean of different cortical regions that are shown separately in $\mathbf{b}$ and $\mathbf{d}$, respectively. Two way ANOVA followed by Bonferroni's posthoc test. Mean + SEM; $N=5 .{ }^{*} p<0.05 ;{ }^{* *} p<0.01 ;{ }^{* * *} p<0.001$. Solid lines: Comparison of AD stages within a brain region; dotted lines: comparison of brain regions of the same AD Braak stage. CiCtx: cingulate cortex; FrCtx: frontal cortex; OcCtx: occipital cortex; TeCtx: temporal cortex; TEntR: transentorhinal region

Braak stage V/VI Ser202/Thr205 tau phosphorylation significantly increased about 4- to 13-fold above control levels (Fig. 2a, b). At Braak stage V/VI the highest Ser202/ Thr205 tau phosphorylation could be observed in the temporal cortex while the lowest phosphorylation was in the cingulate cortex (Fig. 2b). Analysis of the allocortical TEntR showed a first but not significant pSer202/Thr205 tau increase in Braak stage III/IV that became significant at Braak stage V/VI (Fig. 2a).

\section{pThr231 tau}

Phosphorylation of tau at Thr231 was also low in all isocortical brain regions up to Braak stage III/IV. Only at Braak stage V/VI Thr231 tau phosphorylation significantly increased about 30 - to 160-fold above control levels (Fig. 2c, d). At Braak stage V/VI the highest Thr231 tau phosphorylation could be observed in the temporal cortex while the lowest phosphorylation was in the frontal cortex (Fig. 2d). Analysis of the allocortical TEntR showed a first but not significant pThr231 tau increase at Braak stage I/ II that became significant compared to healthy controls at Braak stage III/IV and further significantly increased at Braak stage V/VI compared to all other groups (Fig. 2c).

\section{pSer199 tau}

Overall, phosphorylation of tau at Ser199 was low compared to other phosphorylation sites. In all isocortical regions Ser199 tau phosphorylation started late, namely at Braak stage V/VI (Fig. 3a, b). At Braak stage V/VI Ser199 tau phosphorylation was at comparable levels in all isocortical regions and about 50- (occipital cortex) to 1300-fold (cingulate cortex) increased compared to healthy controls (Fig. 3b). Analysis of the allocortical TEntR showed a progressive increase in Ser199 tau phosphorylation starting at Braak stage I/II but differences between Braak stages became significant only at stage V/IV and were then about 3.5-fold compared to Braak stage III/IV and 160-fold compared to healthy controls (Fig. 3a). Phosphorylation of Ser199 tau in the TEntR of late Braak stages was higher compared to all isocortical regions.

\section{pTyr18 tau}

The phosphorylation profile of pTyr18 tau in the isocortex and TEntR was very similar to pSer199 tau although the final phosphorylation levels in Braak stage V/VI were slightly higher and increased about 20- to 140-fold above control levels (Fig. 3c, d). The progression in the TEntR was already significant at Braak stage III/IV compared to 

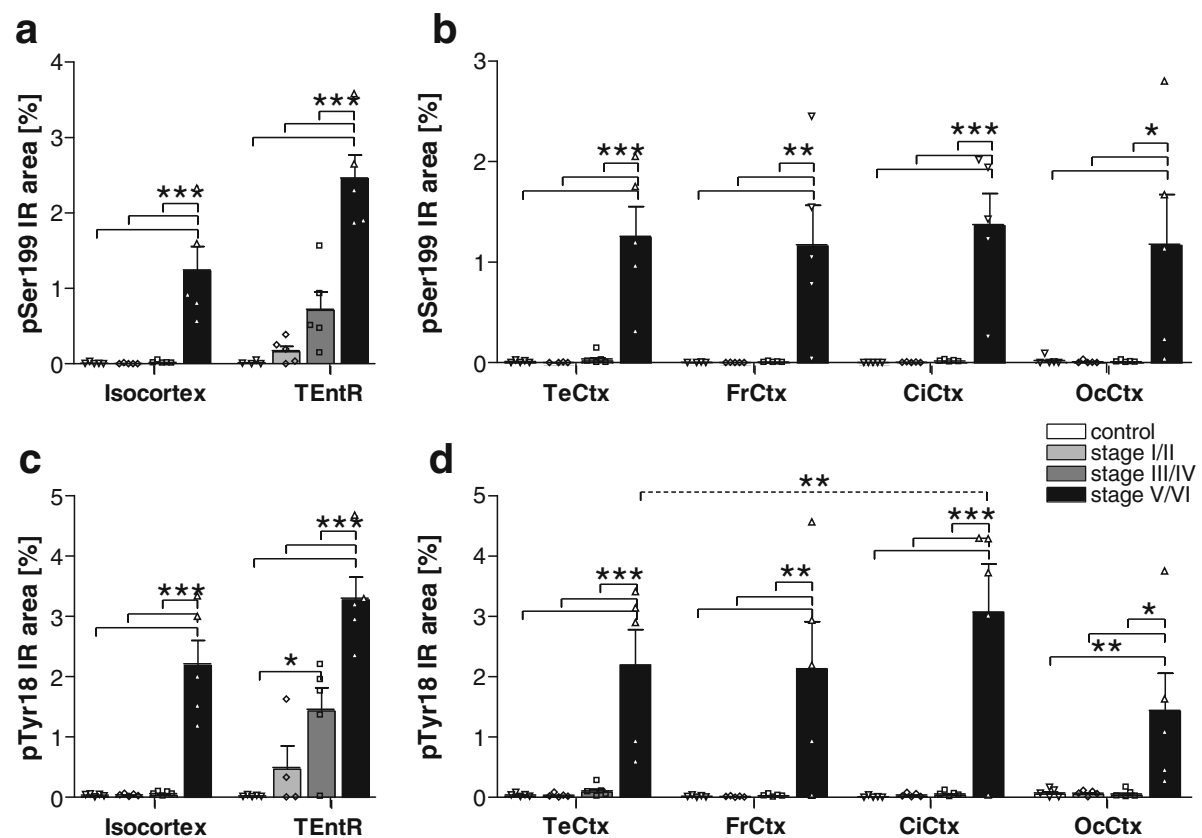

Fig. 3 Quantification of tau phosphorylation at pSer199 and pTyr18 in the cortex of AD cases. a Human pSer199 tau immunoreactive area in percent in the isocortex and TENtR (allocortex) of different AD stages. c Human pTyr18 tau immunoreactive area in percent in the isocortex and TEntR of different AD stages. Isocortical data in $\mathbf{a}$ and $\mathbf{c}$ present mean of different cortical regions that are shown separately in $\mathbf{b}$ and $\mathbf{d}$, respectively. Two way ANOVA followed by Bonferroni's posthoc test. Mean + SEM; $N=5 .{ }^{*} p<0.05$; ${ }^{* *} p<0.01$; ${ }^{* * *} p<0.001$. Solid lines: Comparison of $\mathrm{AD}$ stages within a brain region; dotted lines: comparison of brain regions of the same AD Braak stage. CiCtx: cingulate cortex; FrCtx: frontal cortex; OcCtx: occipital cortex; TeCtx: temporal cortex; TEntR: transentorhinal region

stage I/II and controls. At Braak stage V/VI the highest pTyr18 tau could be observed in the TEntR and cingulate cortex while the lowest phosphorylation was measured in the occipital cortex (Fig. 3c, d).

\section{pSer396 tau}

Phosphorylation of tau at Ser396 shows higher variability than other ptau sites. In the isocortex overall Ser396 tau phosphorylation starts very late in Braak stage V/VI (Fig. 4b). When analyzing different isocortical regions separately, it seemed that pSer396 tau was slightly increased in the temporal cortex of healthy controls and in the cingulate cortex at early Braak stage I/II but these variations were not significant (Fig. 4b). Otherwise, Ser396 tau phosphorylation in all investigated regions only increased about 2- to 5-fold at Braak stage V/VI compared to control levels. Due to the high inter-individual variation this difference was only significant in the temporal cortex compared to Braak stage I/II and III/IV (Fig. 4b). In the allocortical TEntR pSer396 tau increased significantly at Braak stage V/VI compared to all other groups (Fig. 4a).

\section{pSer262 tau}

Phosphorylation of tau at Ser262 was very low and showed almost no progression in higher Braak stages (Fig. 4c). Only in the TEntR a significant 8-fold increase could be noted between Braak stage I/II and V/VI (Fig. 4d).

\section{pSer422 tau}

The phosphorylation profile of tau at Ser422 was comparable in all isocortical regions and also the allocortical TEntR. In all analyzed regions phosphorylation significantly increased at Braak stage V/VI (Fig. 4e, f) and was about 3- to 13-fold higher compared to the control group.

\section{Relative signal increase of Thios and ptau}

When evaluating the signal increase relative to healthy control brain tissue, it became evident that ThioS labeling in the TEntR increased already at Braak stage III/IV and reached a plateau at Braak stages III/IV and V/VI. The phosphorylation at tau Tyr18 and tau Ser199 severely increased at the same time at Braak stage V/VI with already a slight increase at Braak stage III/IV (Fig. 5a). Compared to this tau phosphorylation the changes at other sites were only minor. Analysis of the phosphorylation relative to healthy control tissue in isocortical brain regions (Fig. 5b-e) showed a progressive increase of Thios labeling in all regions with highest values in the temporal cortex (Fig. 5b). Additionally, tau 

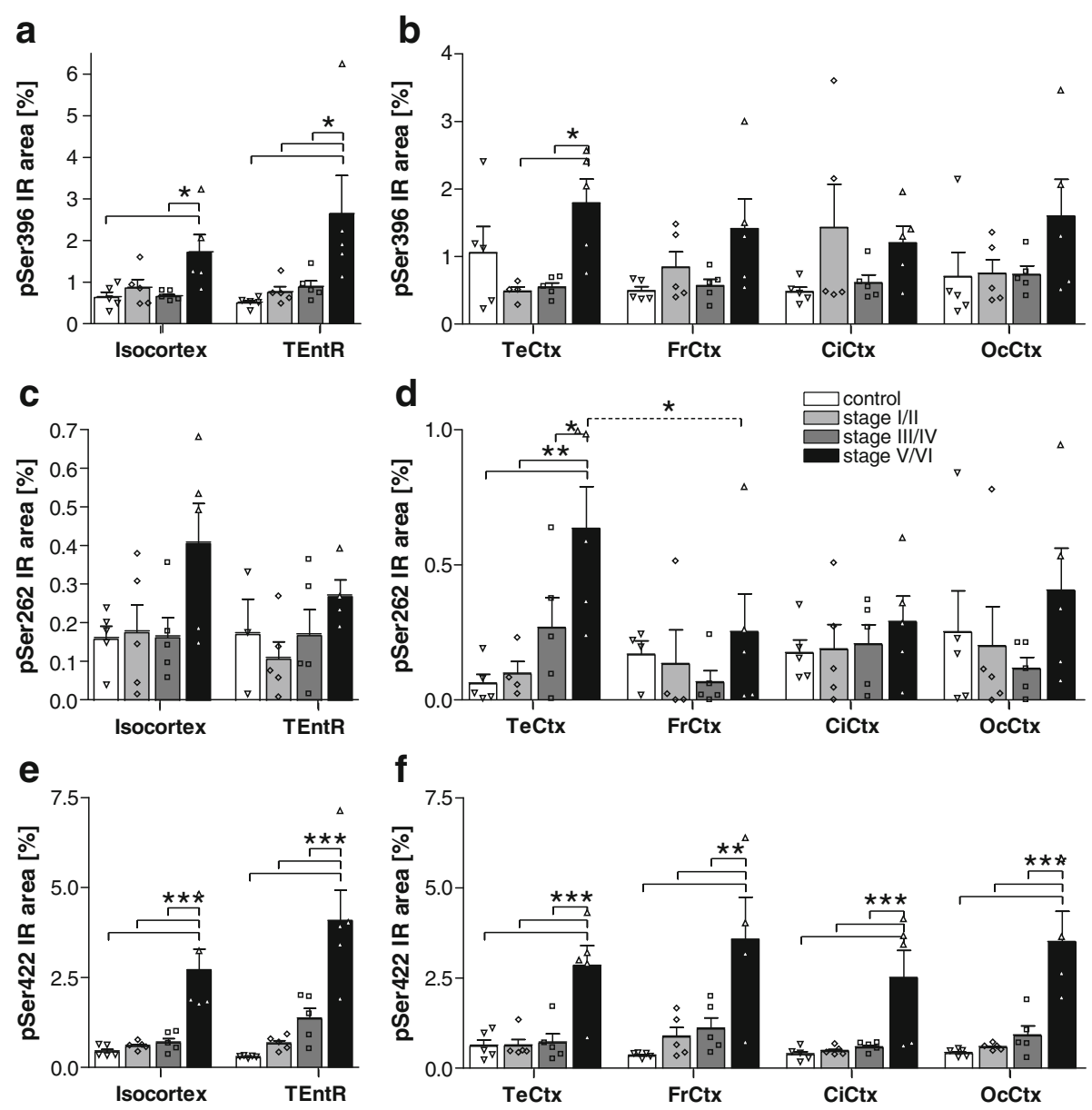

Fig. 4 Quantification of tau phosphorylation at pSer396, pSer262 and pSer422 in the cortex of AD cases. a Human pSer396 tau immunoreactive area in percent in the isocortex and TEntR (allocortex) of different AD stages. c Human pSer422 tau immunoreactive area in percent in the isocortex and TEntR of different AD stages. e Human pSer262 tau immunoreactive area in percent in the isocortex and TEntR of different AD stages. Isocortical data in $\mathbf{a}, \mathbf{c}$ and $\mathbf{e}$ present mean of different cortical regions that are shown separately in $\mathbf{b}, \mathbf{d}$ and $\mathbf{f}$, respectively. Two way ANOVA followed by Bonferroni's posthoc test. Mean + SEM; $N=5 .{ }^{*} p<0.05 ;{ }^{* *} p<0.01 ;{ }^{* * *} p<0.001$. Solid lines: Comparison of AD stages within a brain region; dotted lines: comparison of brain regions of the same AD Braak stage. CiCtx: cingulate cortex; FrCtx: frontal cortex; OcCtx: occipital cortex; TeCtx: temporal cortex; TEntR: transentorhinal region

phosphorylation was severely increased at Thr231, Ser199 and Tyr18. Tau phosphorylation at Thr231 was highest in the temporal cortex (Fig. 5b) while phosphorylation at Ser199 and Tyr18 was highest in the frontal cortex (Fig. 5c). Also in the isocortex tau phosphorylation changes at other sites are only minor compared to Thr231, Ser199 and Tyr18.

To validate results obtained by immunofluorescent labeling, Western blots of $\mathrm{AD}$ and control cases were performed (Fig. 6a). Cases showing an increase in total tau also showed a signal increase in all analyzed ptau residues. Furthermore, the majority of human cases with strong ptau signals were clinically diagnosed with mild dementia (case 6 and 12) or dementia (case 17, 18, 19, Table 1), suggesting a correlation between phosphorylation and cognitive status. Even that tissue of some cases was missing the quantification of Western blot results shows an overall conformity compared to immunofluorescent labelings. Total tau by HT7 labeling (Fig. 6b), pSer202/205 (Fig. 6c), and pSer199 (Fig. 6e) also showed a late signal increase in Braak stage V/VI with only weak signals in earlier Braak stages. Western blotting of pThr231 (Fig. 6d) and pTyr18 (Fig. 6f) also showed a late signal increase in Braak stage V/VI while signals of immunofluorescent labelings increased already in earlier Braak stages (Fig. 2c and Fig. 3c).

Representative images of total tau (Fig. 7) and pSer202/Thr205, pThr231, pSer199 and pTyr18 (Fig. 8) immunofluorescent labelings in the transentorhinal cortex of one case per Braak stage show the progressive increase of these ptau sites.

In summary, our quantitative analysis of $\mathrm{AD}$ brain tissue shows that a progressive increase of total tau and ptau expression can be observed in the 


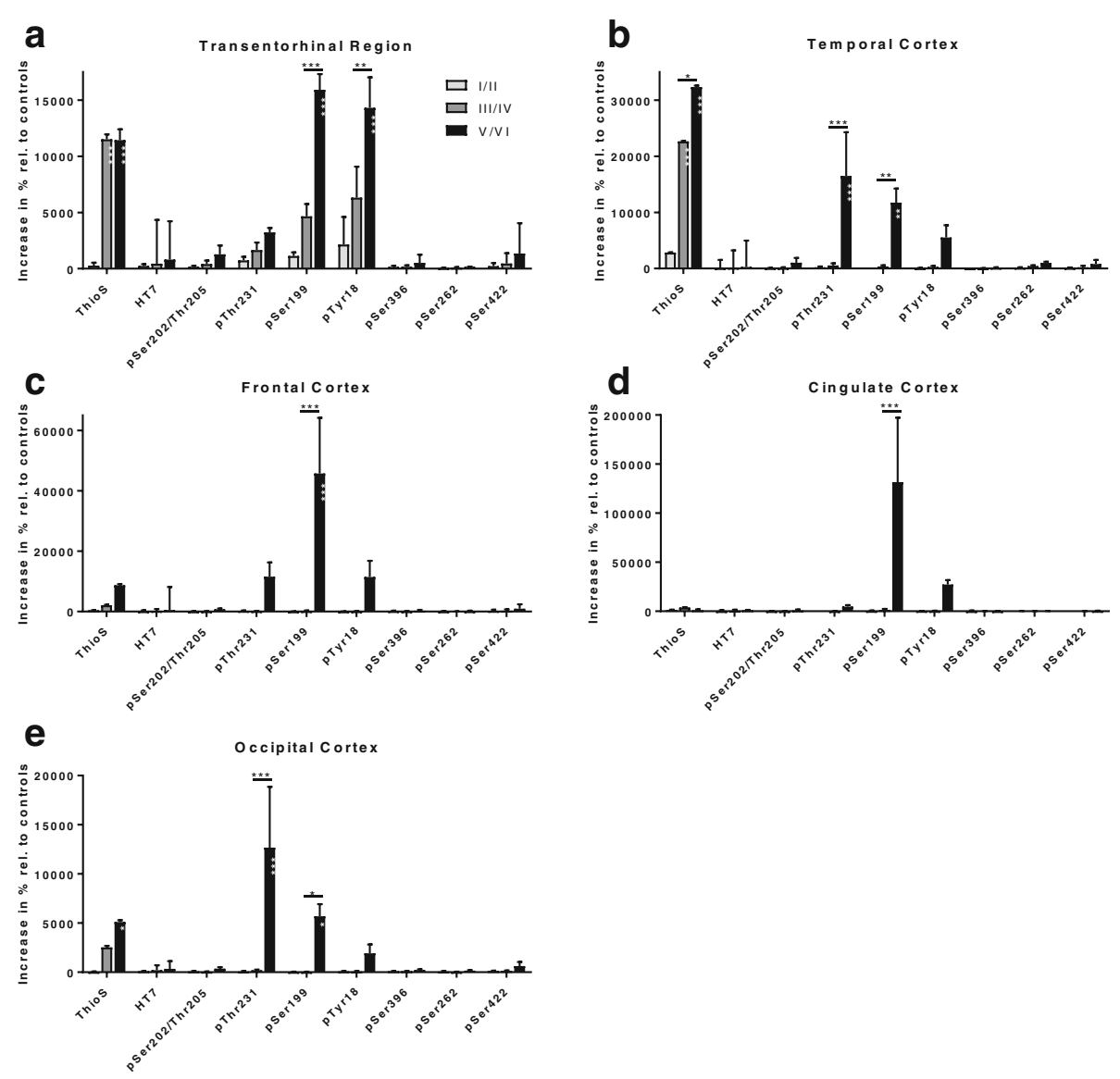

Fig. 5 Signal increase relative to control brain tissue in the cortex of AD cases. Values are calculated from means of absolute IR area from Figs. 1, 2, 3 and 4 relative to control brain samples. Calculation is shown for ThioS, HT7, pSer202/Thr205, pThr231, pSer199, pTyr18, pSer396, pSer262 and pSer422 in the transentorhinal (a), temporal (b), frontal (c), cingulate (d) and occipital cortex (e) at Braak stages I/II; III/IV and VNI. Two way ANOVA followed by Bonferroni's posthoc test. Mean + SEM. White asterisks within bars mark significance compared to Braak stage $1 / 1 \mathrm{Il} .{ }^{*} p<0.05$; ${ }^{* *} p<0.01 ;{ }^{* * *} p<0.001$

transentorhinal region and in most of the analyzed isocortical regions. Staining of tissue with ThioS resulted only in a weak signal in the transentorhinal region and the occipital cortex. Labeling with antibodies against different tau phosphorylation sites showed that most ptau sites, namely pSer202/Thr205, pThr231, pSer199, pTyr18 and pSer422 were highly increased throughout the cortex at Braak stage V/VI. Only tau phosphorylation at Thr231 and Thy18 was already significantly increased at Braak stage III/IV. By contrast, pSer396 tau and pSer262 tau are only weakly expressed in all analyzed brain regions and only minor progression was observed. When comparing tau phosphorylation in percent relative to healthy controls, phosphorylation is specifically increased at tau Thr231, Ser199 and Tyr18. These data indicate that tau phosphorylation is a complex feature of $\mathrm{AD}$ progression, involving many but not all potential phosphorylation sites.

\section{Discussion}

The current study was designed to analyze spatial patterns of tau phosphorylation at multiple residues in discrete anatomical regions during $\mathrm{AD}$ progression. Our data revealed a very similar phosphorylation profile of most of the analyzed ptau sites in the allo- and isocortex while expression levels of phosphorylated tau at Tyr18 and Thr231 was distinguishable between Braak stages. When normalized to controls, phosphorylation of tau at Tyr18, Ser231 and also Ser199 was much more increased at Braak stage V/VI compared to other residues, suggesting a relevance of these sites for $\mathrm{AD}$ progression and a crucial role in pathogenesis.

Since tau phosphorylation is a main characteristic of AD progression, several groups have already analyzed the temporal phosphorylation pattern of different tau sites by histological methods. According to Luna-Munoz and colleagues the phosphorylation of Thr231 tau is an early event in the neuronal pathology of $\mathrm{AD}$ [27]. The 


\section{a}
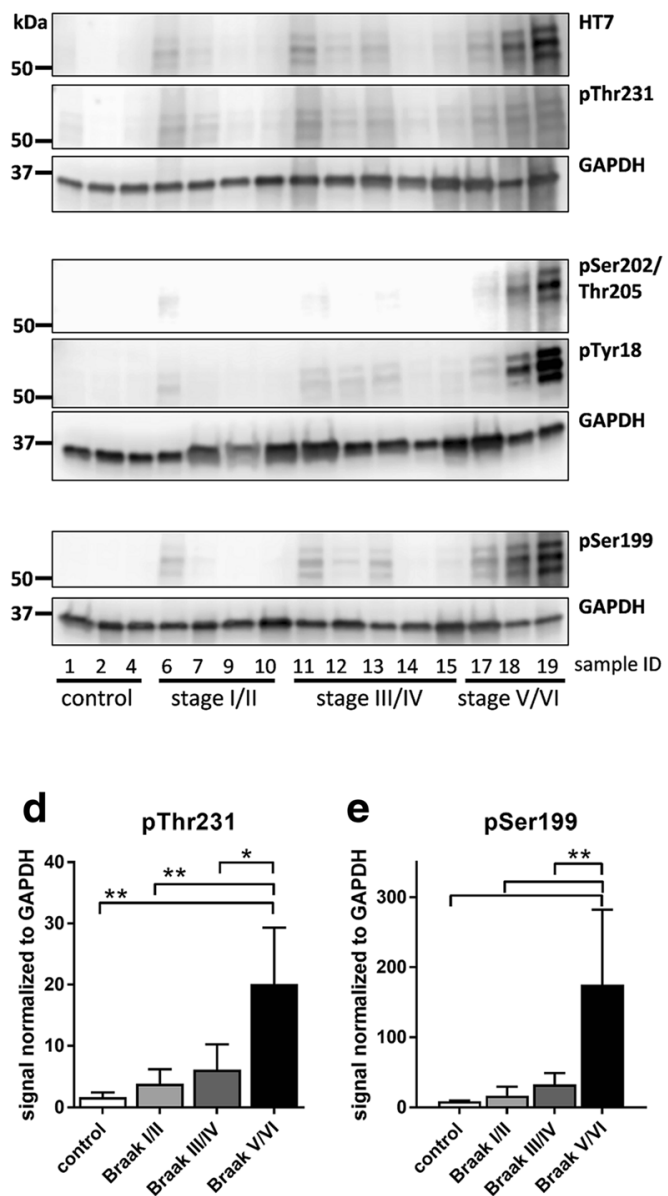

b
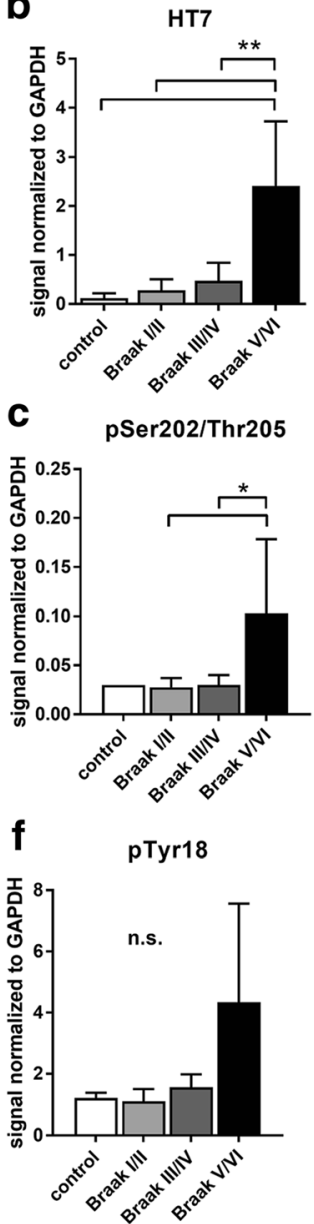

Fig. 6 Quantification of total tau and tau phosphorylation in the transentorhinal cortex of AD cases by Western blotting. a: Western blots of total tau, pThr231, pSer202/Thr205, pTyr18, and pSer199. GAPDH was used as loading control. Quantification of Western blot for (b) total tau by HT7 antibody, (c) pSer202/Thr205, (d) pThr231, (e) pSer199, and (f) pTyr18. All samples shown in (a) were used for quantification of (b-f). One way ANOVA followed by Tukey's multiple comparisons test. Mean + SEM. ${ }^{*} p<0.05 ;{ }^{* *} p<0.01$; n.s.: not significant

temporal analysis of pSer202/Thr205 tau and pSer396 tau is very controversial. Simic and co-workers found higher phosphorylation of Ser396 and Ser202/Thr205 tau in mild cognitive impairment (MCI) cases [38] suggesting a parallel phosphorylation of both residues. Temporal phosphorylation analyses by two other groups contradict these results, while one group found an earlier phosphorylation of pSer202/Thr205 tau [39], the other reported an earlier phosphorylation of pSer396 tau [30]. Zhou and colleagues performed dot blots and ELISA analyses of a whole series of ptau sites of $\mathrm{AD}$ medial temporal cortex samples and found mostly a similar temporal phosphorylation pattern as shown here for the entorhinal cortex. Tau phosphorylation of Ser396 for example, was a late event and only measurable in Braak stage V and VI [46]. Dot blot analyses of AD lateral temporal lobe samples by another group showed that tau phosphorylation at Ser202/205 and Ser396 simultaneously increases with increasing Braak staging, but also that they observe the increase already in Braak stage III/IV compared to results shown here or by Zhou and colleagues [20,46]. By quantitatively analyzing ptau sites in different brain regions of $\mathrm{AD}$ cases we found that most analyzed ptau sites, pSer202/Thr205, pThr231, pSer199, pTyr18 and pSer422, have a very similar alloand isocortical phosphorylation profile, suggesting that pSer202/Thr205 tau analysis by AT8 antibody could be replaced by any of these ptau sites when using this quantitative histological approach. For neuropathological assessment of Braak stages ptau Ser202/Thr205 immunopositivity is scored semi-quantitatively and is required to be mild (+) in the transentorhinal cortex to assign Braak stage I, while for stages higher than Braak stage I, severity of ptau Ser202/Thr205 immunopositivity in the transentorhinal cortex is not decisive as other areas, e.g. entorhinal cortex, occipitotemporal gyrus, 

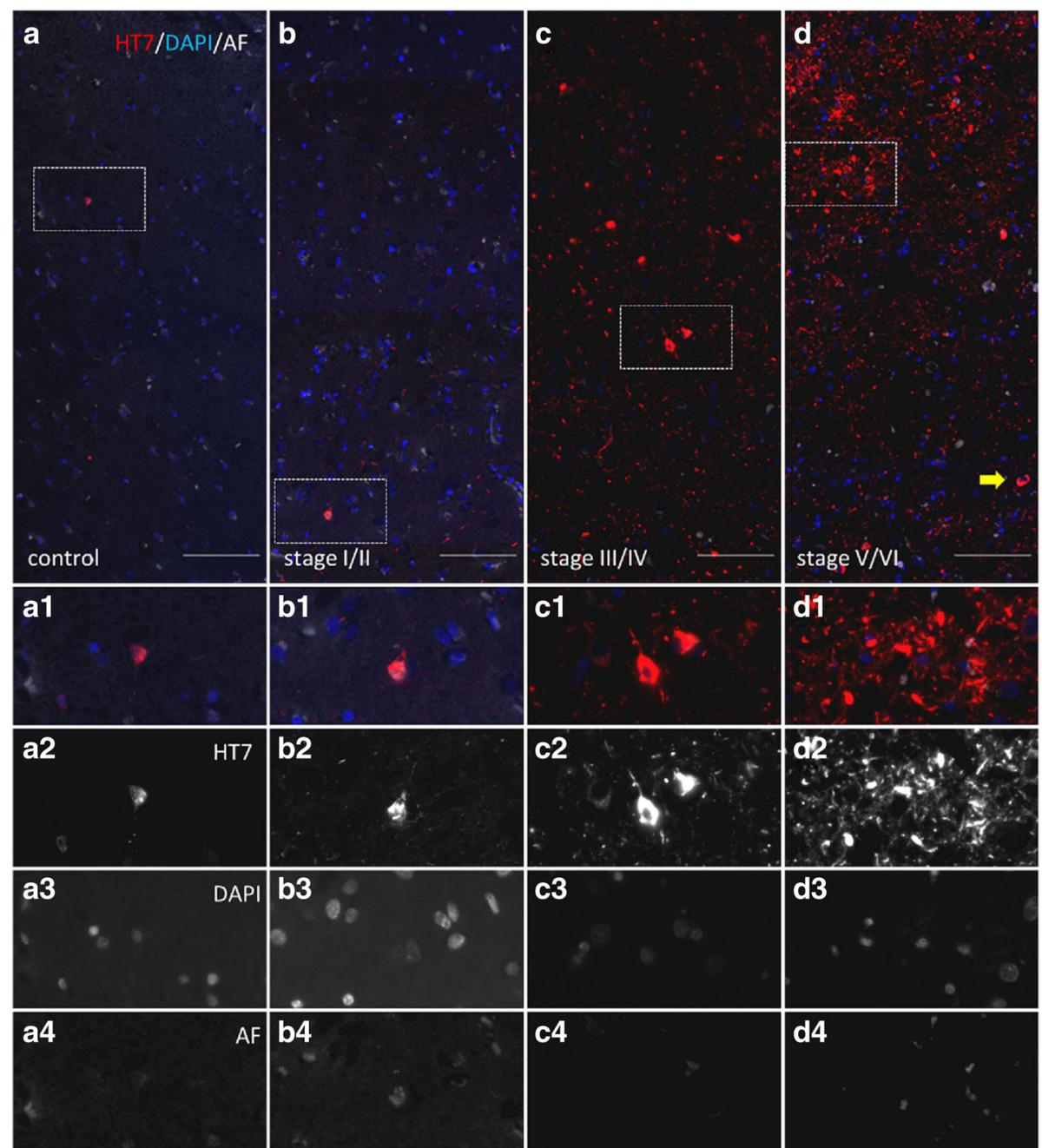

Fig. 7 Representative images of total tau labeling in the transentorhinal cortex grey matter of AD cases. Labeling of total tau using HT7 antibody (a-d, a1-d1, a2-d2) and nuclei by DAPI staining (a-d, a1-d1,a3-d3). Autofluorescence is shown in white (a-d, a1-d1, a4-d4). Samples of healthy control tissue (a-a4, case 2), Braak stage I/II (b-b4, case 8), Braak stage III/IV (c-c4, case 11) and Braak stage V/NI (d-d4, case 16) are shown. Scale bar: $100 \mu \mathrm{m}$

middle temporal gyrus, occipital cortex need to show at least moderate $(++)$ severity of immunopositivity [1]. Therefore our findings of a lack of a significant increase of ptau Ser202/Thr205 immunopositivity in the transentorhinal cortex between Braak stages 0 to IV is not at variance with neuropathological Braak staging. However, pSer202/Thr205 immunopositivity does indeed increase continuously in the transentorhinal cortex with increasing Braak stages. In our study the difference is only statistically significant between Braak stages 0-IV and V/VI. The phosphorylation of the analyzed tau sites starts in the isocortical regions late at Braak stage V/VI and consequently the analysis of the allocortical transentorhinal region is preferable. Indeed, the quantitative analysis of pTyr18 tau and pThr231 tau in the transentorhinal region shows a distinct progression between Braak stages that might be caused by highly increased phosphorylation relative to control tissue that could also be observed for pSer199 tau. Since phosphorylation of tau at residues Tyr18 and Thr231 occurs earlier compared to other sites, these phosphorylation sites might trigger disease progression and thus would represent an early therapeutic target.

Western blot analyses of total tau and the most prominent phosphorylation residues pSer202/Thr205, pThr231, pSer199, and pTyr18 overall validated the results obtained by immunofluorescent labeling. It has to be mentioned that tissue of not all human cases was available for blotting, so quantification and statistical analyses should be understood as preliminary. Blots for pSer202//Thr205 and pThr231 are not directly comparable, since different antibodies as used 

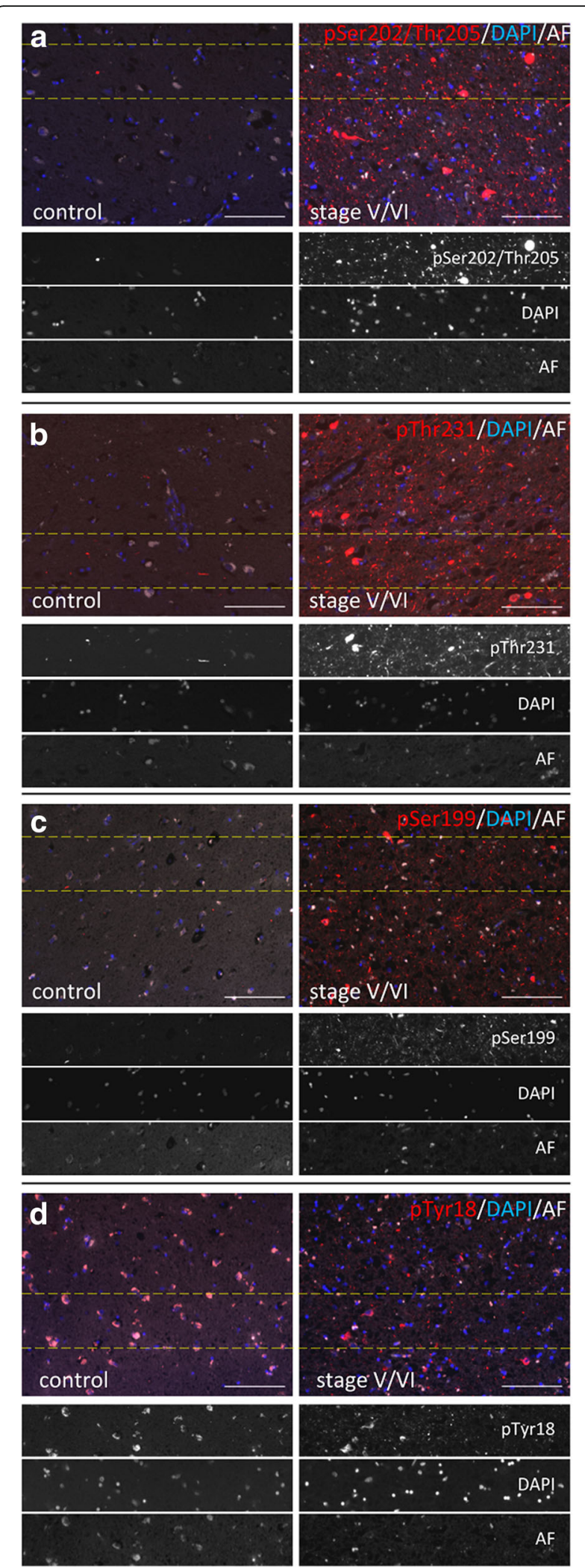

Fig. 8 Representative images of ptau labelings in the transentorhinal cortex of control and AD cases. Labeling of pSer202/Thr205 (a), pThr231 (b), pSer199 (c), and pTyr18 (d) are shown. Tissues were additionally stained with DAPI to visualize nuclei. Autofluorescence is shown in white. Dotted lines indicate areas shown in grey scale images. Samples of healthy control tissue (case 2) and Braak stage V/ VI (case 16) are shown. Scale bar: $100 \mu \mathrm{m}$

for immunofluorescent labelings had to be used for methodical reasons.

$\mathrm{AD}$ is known to affect women with a higher prevalence, which is among other reasons caused by hormonal differences. The higher frequency of $\mathrm{AD}$ cases in women is reflected in this study by the fact that only tissue of women was provided for Braak stage V/VI, while tissue of earlier Braak stages was predominantly from men. Although several in vitro and in vivo studies in rodents suggest an impact of sex hormones (e.g. estrogens, progesterone and prolactin) and therefore gender on tau phosphorylation, there are so far no studies in AD patients validating these results in humans (for review see [33]). Future analyses of post mortem AD tissue and in vivo imaging of $\mathrm{AD}$ patients should shed light on the impact of gender on tau phosphorylation.

In addition to the severe increases in tau phosphorylation also the ThioS signal, that includes amyloid plaques, was highly increased in several allo- and isocortical regions. The ThioS signal increase partially already started at Braak stage III/IV and thus earlier than the increase of ptau. It has been shown that ptau residues, Tyr18, Thr231 and Ser199 can be phosphorylated by $A \beta$ via different kinases like Fyn [18, 35, 37, 42, 44, 45], GSK-3 $\beta$ [3, 23, 24] or CDK5 $[6,25]$. The activation of tau by $A \beta$ is further demonstrated to be involved in the early formation of neurofibrillary tangles, synaptic loss, neurodegeneration as well as cognitive deficits $[2,3,11,25]$ and thus in the development of the most prominent $\mathrm{AD}$ pathologies. These results were derived from $\mathrm{AD}$ cell and animal models but our study might suggest a similar effect of $A \beta$ on tau phosphorylation at residues Tyr18, Thr231 and Ser199 in the human disease. Further analyses are needed to validate this hypothesis in humans. A valid tool to analyze such events in vivo might be the use of Pittsburgh compound B (PiB) analysis combined with tau tracer that are currently under development $[5,17,19,36]$.

Isocortical tau pathology is only sparse at early Braak stages I to IV and hence use of higher magnification and modified image analysis parameters may be necessary to reveal subtle changes in isocortical tau pathology at early Braak stages. Additionally, co-labeling of different markers to quantify ptau only in distinct areas or cell populations combined with an increased number of investigated brain regions may be helpful to gain a better understanding of earliest isocortical tau pathology. A 
tissue microarray method that was developed recently allows to examine over 35 brain regions on one slide could be used for such purposes [43]. However, our study investigated only five brain regions in a limited number of cases and represents therefore proof of principle. Our data need to be confirmed by including more cases and assessing a higher number of brain areas.

\section{Conclusion}

We show here for the first time the phosphorylation profile of different tau sites in allocortical and isocortical regions of human brains with tau pathology ranging from Braak stages 0 to VI using quantitative rater-independent immunofluorescent labeling. Our data suggest that the profile of ptau in the isocortex is comparable between all of the analyzed ptau sites while expression levels of ptau at Thr231 and Tyr18 in the transentorhinal region are distinguishable between Braak stages combined with the highest tau phosphorylation relative to controls at these sites and Ser199.

\section{Additional file}

Additional file 1: Online Source 9 Double labeling of pSer262 and pSer202/Thr205 tau in the temporal cortex at Braak stage VNI. Images show different labeling pattern of pSer262 (arrows) and pS202 (white arrowheads) as well as their overlay (yellow arrowheads) (a1) and single fluorescence images $(a 2,3,4)$ of case 17. AF: autofluorescence. Scale bar: $20 \mu \mathrm{m}$. Online Source 10 Example of measurement procedure of tau pSer262. Objects in the unlabeled autofluorescence channel were detected by thresholding (red in a1). The resulting mask images (a2) were then subtracted from tau pSer262 images to remove autofluorescence (a3). The resulting images were Edge+ filtered (a4) to facilitate threshold-based detection of tau pSer262-positive objects (red outline in a5). These outlines were then loaded onto the raw images to quantify original tau pSer262 signal (red outline in a6). AF: autofluorescence. Scale bar: $20 \mu \mathrm{m}$. Online Source 11 Example of detecting ThioS-positive amyloid- $\beta$ but not NFTs. Image a displays the co-labeling of ThioS (green) and HT7 (red), while images b and c, respectively, show single channel images. ThioS shows intense labeling of plaque-associated $\beta$-sheets ( $b$, asterisk) whereas tangles are only weakly labeled (c, arrows) (c). A combination of threshold-based identification of ThioS and size restriction ( $d$ ', green rectangle) enables quantification of ThioS+ plaque labeling (red highlighted) but not tangles (d). Thios: Thioflavins. Scale bar: 20 m. (PDF 599 kb)

\section{Abbreviations}

AD: Alzheimer's disease; ANOVA: Analysis of variance; CiCtx: Cingulate cortex; FrCtx: Frontal cortex; NFT: Neurofibrillary tangle; NT: Neuropil threads; OcCtx: Occipital cortex; ptau: Phosphorylated tau; TeCtx: Temporal cortex; TEntR: Transentorhinal region

\section{Acknowledgements}

The authors greatly thank the whole research team of QPS Austria GmbH for their technical support.

\section{Funding}

This work was supported by the Austrian Research Promotion Agency (FFG), R\&D Projects (FFG\# 844453, 851079, 855287).

Tissue for this study was provided by the Newcastle Brain Tissue Resource, which is funded in part by a grant from the UK Medical Research Council (G0400074), by Brains for Dementia research, a joint venture between Alzheimer's Society and Alzheimer's Research UK and by the NIHR Newcastle
Biomedical Research Centre awarded to the Newcastle upon Tyne Hospitals NHS Foundation Trust and Newcastle University.

\section{Availability of data and materials}

The datasets used and/or analyzed during the current study are available from the corresponding author on reasonable request.

\section{Authors' contributions}

JN designed the overall project, analyzed data, interpreted experiments and prepared the manuscript; MT analyzed data and prepared figures; SF interpreted experiments and prepared figures and the manuscript; BK and $\mathrm{CH}$ performed experiments and analyzed data; VN edited the manuscript; JA provided neuropathologically diagnoses and edited the manuscript; GD interpreted experiments and edited the manuscript; BHP designed and interpreted experiments and edited the manuscript. All authors read and approved the final manuscript.

\section{Ethics approval and consent to participate}

Human tissue was provided by the Newcastle Brain Tissue Resource (NBTR), Newcastle University, UK in accordance with the approval of the joint Ethics Committee of Newcastle and North Tyneside Health Authority and following NBTR brain banking procedures.

\section{Consent for publication}

Not applicable.

\section{Competing interests}

JN, MT, SF, BK, CH, TL, VN and BHP are employees of QPS Austria GmbH. The authors declare that they have no other competing interests.

\section{Publisher's Note}

Springer Nature remains neutral with regard to jurisdictional claims in published maps and institutional affiliations.

\section{Author details}

${ }^{1}$ QPS Austria GmbH, Neuropharmacology, Parkring 12, 8074 Grambach, Austria. ${ }^{2}$ Institute for Biochemistry, Graz University of Technology, Graz, Austria. Institute of Zoology, Karl Franzens University, Graz, Austria. ${ }^{4}$ Institute of Neuroscience and Newcastle University Institute for Ageing Campus for Ageing and Vitality, Newcastle University, Newcastle upon Tyne NE4 5PL, UK.

Received: 24 May 2018 Accepted: 19 June 2018

Published online: 29 June 2018

\section{References}

1. Alafuzoff I, Arzberger T, Al-Sarraj S, Bodi I, Bogdanovic N, Braak H, Bugiani O, Del-Tredici K, Ferrer I, Gelpi E et al (2008) Staging of neurofibrillary pathology in Alzheimer's disease: a study of the BrainNet Europe consortium. Brain Pathol 18: 484-496 doi 10.1111/j.1750-3639.2008.00147.x

2. Amadoro G, Corsetti V, Atlante A, Florenzano F, Capsoni S, Bussani R, Mercanti D, Calissano P (2012) Interaction between NH(2)-tau fragment and Abeta in Alzheimer's disease mitochondria contributes to the synaptic deterioration. Neurobiol Aging 33(833):e831-e825. https://doi.org/10.1016/j. neurobiolaging.2011.08.001

3. Amadoro G, Corsetti V, Ciotti MT, Florenzano F, Capsoni S, Amato G, Calissano P (2011) Endogenous Abeta causes cell death via early tau hyperphosphorylation. Neurobiol Aging 32:969-990. https://doi.org/10.1016/ j.neurobiolaging.2009.06.005

4. Andorfer C, Kress Y, Espinoza M, de Silva R, Tucker KL, Barde YA, Duff K, Davies $P$ (2003) Hyperphosphorylation and aggregation of tau in mice expressing normal human tau isoforms. J Neurochem 86:582-590

5. Ariza M, Kolb HC, Moechars D, Rombouts F, Andres JI (2015) Tau positron emission tomography (PET) imaging: past, present, and future. J Med Chem 58:4365-4382. https://doi.org/10.1021/jm5017544

6. Billingsley ML, Kincaid RL (1997) Regulated phosphorylation and dephosphorylation of tau protein: effects on microtubule interaction, intracellular trafficking and neurodegeneration. Biochem J 323(Pt 3):577-591

7. Boekhoorn K, Terwel D, Biemans B, Borghgraef P, Wiegert O, Ramakers GJ, de Vos K, Krugers $\mathrm{H}$, Tomiyama T, Mori $\mathrm{H}$ et al (2006) Improved long-term potentiation and memory in young tau-P301L transgenic mice before onset 
of hyperphosphorylation and tauopathy. J Neurosci 26:3514-3523. https:// doi.org/10.1523/JNEUROSCI.5425-05.2006

8. Braak H, Alafuzoff I, Arzberger T, Kretzschmar H, Del Tredici K (2006) Staging of Alzheimer disease-associated neurofibrillary pathology using paraffin sections and immunocytochemistry. Acta Neuropathol 112:389-404. https:// doi.org/10.1007/s00401-006-0127-z

9. Braak H, Braak E (1991) Neuropathological stageing of Alzheimer-related changes. Acta Neuropathol 82:239-259

10. Bramblett GT, Goedert M, Jakes R, Merrick SE, Trojanowski JQ, Lee VM (1993) Abnormal tau phosphorylation at Ser396 in Alzheimer's disease recapitulates development and contributes to reduced microtubule binding. Neuron 10:1089-1099

11. Chang KH, de Pablo Y, Lee HP, Lee HG, Smith MA, Shah K (2010) Cdk5 is a major regulator of p38 cascade: relevance to neurotoxicity in Alzheimer's disease. J Neurochem 113:1221-1229. https://doi.org/10.1111/j.1471-4159. 2010.06687.x

12. Cho JH, Johnson GV (2004) Primed phosphorylation of tau at Thr231 by glycogen synthase kinase 3beta (GSK3beta) plays a critical role in regulating tau's ability to bind and stabilize microtubules. J Neurochem 88:349-358

13. Cook C, Stankowski JN, Carlomagno Y, Stetler C, Petrucelli L (2014) Acetylation: a new key to unlock tau's role in neurodegeneration. Alzheimers Res Ther 6:29. https://doi.org/10.1186/alzrt259

14. Flunkert S, Hierzer M, Loffler T, Rabl R, Neddens J, Duller S, Schofield EL, Ward MA, Posch M, Jungwirth $\mathrm{H}$ et al (2013) Elevated levels of soluble total and hyperphosphorylated tau result in early behavioral deficits and distinct changes in Brain Pathol in a new tau transgenic mouse model. Neurodegener Dis 11:194-205. https://doi.org/10.1159/000338152

15. Goedert M, Jakes R, Crowther RA, Six J, Lubke U, Vandermeeren M, Cras P, Trojanowski JQ, Lee VM (1993) The abnormal phosphorylation of tau protein at Ser-202 in Alzheimer disease recapitulates phosphorylation during development. Proc Natl Acad Sci U S A 90:5066-5070

16. Green KN, Steffan JS, Martinez-Coria H, Sun X, Schreiber SS, Thompson LM, LaFerla FM (2008) Nicotinamide restores cognition in Alzheimer's disease transgenic mice via a mechanism involving sirtuin inhibition and selective reduction of Thr231-phosphotau. J Neurosci 28:11500-11510. https://doi. org/10.1523/JNEUROSCI.3203-08.2008

17. Hashimoto H, Kawamura K, Igarashi N, Takei M, Fujishiro T, Aihara Y, Shiomi S, Muto M, Ito T, Furutsuka K et al (2014) Radiosynthesis, photoisomerization, biodistribution, and metabolite analysis of 11C-PBB3 as a clinically useful PET probe for imaging of tau pathology. J Nucl Med 55: 1532-1538. https://doi.org/10.2967/jnumed.114.139550

18. Ittner LM, Ke YD, Delerue F, Bi M, Gladbach A, van Eersel J, Wolfing $H$, Chieng BC, Christie MJ, Napier IA et al (2010) Dendritic function of tau mediates amyloid-beta toxicity in Alzheimer's disease mouse models. Cell 142:387-397. https://doi.org/10.1016/j.cell.2010.06.036

19. Kadir A, Nordberg A (2010) Target-specific PET probes for neurodegenerative disorders related to dementia. J Nucl Med 51:1418-1430. https://doi.org/10.2967/jnumed.110.077164

20. Koss DJ, Jones G, Cranston A, Gardner H, Kanaan NM, Platt B (2016) Soluble pre-fibrillar tau and beta-amyloid species emerge in early human Alzheimer's disease and track disease progression and cognitive decline. Acta Neuropathol 132:875-895. https://doi.org/10.1007/s00401016-1632-3

21. Lauckner J, Frey P, Geula C (2003) Comparative distribution of tau phosphorylated at Ser262 in pre-tangles and tangles. Neurobiol Aging 24:767-776

22. Lee G, Thangavel R, Sharma VM, Litersky JM, Bhaskar K, Fang SM, Do LH, Andreadis A, Van Hoesen G, Ksiezak-Reding H (2004) Phosphorylation of tau by fyn: implications for Alzheimer's disease. J Neurosci 24:2304-2312. https://doi.org/10.1523/JNEUROSCI.4162-03.2004

23. Li T, Hawkes C, Qureshi HY, Kar S, Paudel HK (2006) Cyclin-dependent protein kinase 5 primes microtubule-associated protein tau site-specifically for glycogen synthase kinase 3beta. Biochemistry 45:3134-3145. https://doi. org/10.1021/bi051635j

24. Li T, Paudel HK (2006) Glycogen synthase kinase 3beta phosphorylates Alzheimer's disease-specific Ser396 of microtubule-associated protein tau by a sequential mechanism. Biochemistry 45:3125-3133. https://doi. org/10.1021/bi051634

25. Liao X, Zhang Y, Wang Y, Wang J (2004) The effect of cdk-5 overexpression on tau phosphorylation and spatial memory of rat. Sci China Ser C Life Sci 47:251-257
26. Lichtenberg-Kraag B, Mandelkow EM, Biernat J, Steiner B, Schroter C, Gustke N, Meyer HE, Mandelkow E (1992) Phosphorylation-dependent epitopes of neurofilament antibodies on tau protein and relationship with Alzheimer tau. Proc Natl Acad Sci U S A 89:5384-5388

27. Luna-Munoz J, Garcia-Sierra F, Falcon V, Menendez I, Chavez-Macias L, Mena $R$ (2005) Regional conformational change involving phosphorylation of tau protein at the Thr231, precedes the structural change detected by Alz-50 antibody in Alzheimer's disease. J Alzheimer's Dis: JAD 8:29-41

28. Lund H, Cowburn RF, Gustafsson E, Stromberg K, Svensson A, Dahllund L, Malinowsky D, Sunnemark D (2013) Tau-tubulin kinase 1 expression, phosphorylation and co-localization with phospho-Ser422 tau in the Alzheimer's disease Brain. Brain Pathol 23:378-389. https://doi.org/10.1111/ bpa. 12001

29. Luo HB, Xia YY, Shu XJ, Liu ZC, Feng Y, Liu XH, Yu G, Yin G, Xiong YS, Zeng $K$ et al (2014) SUMOylation at K340 inhibits tau degradation through deregulating its phosphorylation and ubiquitination. Proc Natl Acad Sci U S A 111:16586-16591. https://doi.org/10.1073/pnas.1417548111

30. Mondragon-Rodriguez S, Perry G, Zhu X, Moreira PI, Acevedo-Aquino MC, Williams S (2013) Phosphorylation of tau protein as the link between oxidative stress, mitochondrial dysfunction, and connectivity failure: implications for Alzheimer's disease. Oxidative Med Cell Longev 2013: 940603. https://doi.org/10.1155/2013/940603

31. Montine TJ, Phelps CH, Beach TG, Bigio EH, Cairns NJ, Dickson DW, Duyckaerts C, Frosch MP, Masliah E, Mirra SS et al (2012) National Institute on Aging-Alzheimer's Association guidelines for the neuropathologic assessment of Alzheimer's disease: a practical approach. Acta Neuropathologica 123:1-11. https://doi.org/10.1007/ s00401-011-0910-3

32. Moszczynski AJ, Gohar M, Volkening K, Leystra-Lantz C, Strong W, Strong MJ (2015) Thr175-phosphorylated tau induces pathologic fibril formation via GSK3beta-mediated phosphorylation of Thr231 in vitro. Neurobiol Aging 36: 1590-1599. https://doi.org/10.1016/..neurobiolaging.2014.12.001

33. Munoz-Mayorga D, Guerra-Araiza C, Torner L, Morales T (2018) Tau phosphorylation in female neurodegeneration: role of estrogens, progesterone, and prolactin. Front Endocrinol 9:133. https://doi.org/10.3389/ fendo.2018.00133

34. Pevalova M, Filipcik P, Novak M, Avila J, Iqbal K (2006) Post-translational modifications of tau protein. Bratislavske Lekarske Listy 107:346-353

35. Roberson ED, Halabisky B, Yoo JW, Yao J, Chin J, Yan F, Wu T, Hamto P, Devidze N, Yu GQ et al (2011) Amyloid-beta/Fyn-induced synaptic, network, and cognitive impairments depend on tau levels in multiple mouse models of Alzheimer's disease. J Neurosci 31:700-711. https://doi.org/10.1523/ JNEUROSCI.4152-10.2011

36. Rowe CC, Villemagne VL (2013) Amyloid imaging with PET in early Alzheimer disease diagnosis. Med Clin North Am 97:377-398. https://doi. org/10.1016/j.mcna.2012.12.017

37. Scales TM, Derkinderen P, Leung KY, Byers HL, Ward MA, Price C, Bird IN Perera T, Kellie S, Williamson R et al (2011) Tyrosine phosphorylation of tau by the SRC family kinases Ick and fyn. Mol Neurodegen 6:12. https://doi.org/ 10.1186/1750-1326-6-12

38. Simic G, Babic Leko M, Wray S, Harrington C, Delalle I, Jovanov-Milosevic N, Bazadona D, Buee L, de Silva R, Di Giovanni G et al (2016) Tau protein hyperphosphorylation and aggregation in Alzheimer's disease and other Tauopathies, and possible neuroprotective strategies. Biomolecules 6: 6 doi https://doi.org/10.3390/biom6010006

39. Su JH, Cummings BJ, Cotman CW (1994) Early phosphorylation of tau in Alzheimer's disease occurs at Ser-202 and is preferentially located within neurites. Neuroreport 5:2358-2362

40. Tenreiro S, Eckermann K, Outeiro TF (2014) Protein phosphorylation in neurodegeneration: friend or foe? Front Mol Neurosci 7:42. https://doi.org/ 10.3389/fnmol.2014.00042

41. Thal DR, Rub U, Orantes M, Braak H (2002) Phases of a beta-deposition in the human brain and its relevance for the development of AD. Neurology 58:1791-1800

42. Usardi $A$, Pooler AM, Seereeram A, Reynolds $C H$, Derkinderen $P$, Anderton B, Hanger DP, Noble W, Williamson R (2011) Tyrosine phosphorylation of tau regulates its interactions with Fyn $\mathrm{SH} 2$ domains, but not SH3 domains, altering the cellular localization of tau. FEBS J 278:2927-2937. https://doi.org/10.1111/j.1742-4658.2011.08218.x

43. Walker L, McAleese KE, Johnson M, Khundakar AA, Erskine D, Thomas AJ, McKeith IG, Attems J (2017) Quantitative neuropathology: an update on 
automated methodologies and implications for large scale cohorts. J Neural Transm (Vienna). https://doi.org/10.1007/s00702-017-1702-2

44. Williamson R, Scales T, Clark BR, Gibb G, Reynolds CH, Kellie S, Bird IN, Varndell IM, Sheppard PW, Everall I et al (2002) Rapid tyrosine

phosphorylation of neuronal proteins including tau and focal adhesion kinase in response to amyloid-beta peptide exposure: involvement of Src family protein kinases. J Neurosci 22:10-20

45. Williamson R, Usardi A, Hanger DP, Anderton BH (2008) Membrane-bound beta-amyloid oligomers are recruited into lipid rafts by a fyn-dependent mechanism. FASEB J 22:1552-1559. https://doi.org/10.1096/fj.07-9766com

46. Zhou XW, Li X, Bjorkdahl C, Sjogren MJ, Alafuzoff I, Soininen H, Grundkelqbal I, Iqbal K, Winblad B, Pei JJ (2006) Assessments of the accumulation severities of amyloid beta-protein and hyperphosphorylated tau in the medial temporal cortex of control and Alzheimer's brains. Neurobiol Dis 22: 657-668. https://doi.org/10.1016/j.nbd.2006.01.006

Ready to submit your research? Choose BMC and benefit from:

- fast, convenient online submission

- thorough peer review by experienced researchers in your field

- rapid publication on acceptance

- support for research data, including large and complex data types

- gold Open Access which fosters wider collaboration and increased citations

- maximum visibility for your research: over $100 \mathrm{M}$ website views per year

At BMC, research is always in progress.

Learn more biomedcentral.com/submissions 